Bond University

Research Repository

\title{
Caffeine use and alexithymia in university students
}

\author{
Lyvers, Michael; Duric, Natalija; Thorberg, Fred Arne
}

Published in:

Journal of Psychoactive Drugs

DOI:

10.1080/02791072.2014.942043

\section{Licence:}

CC BY-NC-ND

Link to output in Bond University research repository.

Recommended citation(APA):

Lyvers, M., Duric, N., \& Thorberg, F. A. (2014). Caffeine use and alexithymia in university students. Journal of Psychoactive Drugs, 46(4), 340-346. https://doi.org/10.1080/02791072.2014.942043

\footnotetext{
General rights

Copyright and moral rights for the publications made accessible in the public portal are retained by the authors and/or other copyright owners and it is a condition of accessing publications that users recognise and abide by the legal requirements associated with these rights.
}

For more information, or if you believe that this document breaches copyright, please contact the Bond University research repository coordinator. 
Caffeine Use and Alexithymia in University Students

This is an Accepted Manuscript version of the following article:

Lyvers, M., Duric, N., \& Thorberg, F. A. (2014). Caffeine use and alexithymia in university students. Journal of Psychoactive Drugs, 46(4), 340-346. https://doi.org/10.1080/02791072.2014.942043.

It is deposited under the terms of the Creative Commons Attribution-NonCommercial-NoDerivatives License, which permits non-commercial re-use, distribution, and reproduction in any medium, provided the original work is properly cited, and is not altered, transformed, or built upon in any way. 


\begin{abstract}
Alexithymia refers to difficulties with identifying, describing, and regulating one's own emotions. This trait dimension has been linked to risky or harmful use of alcohol and illicit drugs; however the most widely used psychoactive drug in the world, caffeine, has not been examined previously in relation to alexithymia. The present study assessed 106 male and female university students aged 18-30 years on their caffeine use in relation to several traits including alexithymia. The 18 participants defined as alexithymic based on their Toronto Alexithymia Scale (TAS-20) scores reported consuming nearly twice as much caffeine per day as did non-alexithymic or borderline alexithymic participants. They also scored significantly higher than controls on indices of frontal lobe dysfunction as well as anxiety symptoms and sensitivity to punishment. In a hierarchical linear regression model, sensitivity to punishment negatively predicted daily caffeine intake, suggesting caffeine avoidance by trait anxious individuals. Surprisingly however, TAS-20 alexithymia scores positively predicted caffeine consumption. Possible reasons for the positive relationship between caffeine use and alexithymia are discussed, concluding that this outcome is tentatively consistent with the hypoarousal model of alexithymia.
\end{abstract}

KEYWORDS: caffeine, anxiety, emotions, frontal lobes

This is an Accepted Manuscript version of the following article:

Lyvers, M., Duric, N., \& Thorberg, F. A. (2014). Caffeine use and alexithymia in university students. Journal of Psychoactive Drugs, 46(4), 340-346. https://doi.org/10.1080/02791072.2014.942043. 
Alexithymia is a personality dimension referring to persistent difficulties with identifying, describing, and regulating one's own emotional feelings (Nemiah, Freyberger \& Sifneos, 1976). Alexithymia has been linked to self-reported behavioural signs of frontal lobe dysfunction, impaired ability to self-regulate negative moods, and earlier, heavier and riskier levels of alcohol (Franz et al., 2008; Kauhanen et al., 1992; Lyvers, Onuoha, Thorberg \& Samios, 2012; Thorberg, Young, Sullivan, \& Lyvers, 2009) and illicit drug use (Lyvers, Jamieson \& Thorberg, in press; Troisi, Pasini, Saracco, \& Spalletta, 1998). However, alexithymia has not been examined in relation to consumption of the most popular psychoactive drug in the world: caffeine.

Caffeine, an alkaloid found in tea, coffee, chocolate and other plant sources, is a centrally acting adenosine antagonist that indirectly promotes catecholamine release (Grilly, 2006). The stimulant drug is widely available in various beverages and over-the-counter medications, with an average cup of coffee containing about $100 \mathrm{mg}$ of caffeine (Bryan, 2008).

Although caffeine is commonly ingested to improve alertness and counteract fatigue (Childs \& De Wit, 2006; Van Duinen, Lorist \& Zijdewind, 2005), it can also provoke anxiety symptoms, especially when ingested in relatively high doses of $300 \mathrm{mg}$ or more (Evans \& Griffith, 1992; Lara, 2010; Lieberman, Wurtman, Emde, Roberts, \& Covielle, 1987; Penolazzi et al, 2012). Due to this unpleasant anxiogenic effect, many anxious individuals may learn to consume only low doses or even avoid caffeine entirely (Smith, 2002; Telch, Silverman \& Schmidt, 1996). Alexithymia is commonly associated with elevated levels of trait anxiety and anxiety sensitivity (Berthoz, Consoli, Perez-Diaz \& Jovent, 1999; DeGucht, Fischler \& Heiser, 2004; de Timary, Emmanuel, Luminet, Fillee \& Mikolajczak, 2008; Devine, Stewart \& Watt, 1999; Lyvers, Hasking, Albrecht \& Thorberg, 2012; Stewart, Zvolensky \& Eifert, 2002; Tutkun et al, 2004), thus patterns of caffeine use might be expected to differ substantially from use of other drugs in relation to alexithymia. That is, given their relatively high levels of trait 
anxiety and anxiety sensitivity, those with alexithymia might be expected to avoid high doses of caffeine due to the drug's aversive anxiogenic effects. On the other hand, given the associations of alexithymia with heavy use of other psychoactive substances, a positive relationship between alexithymia and caffeine use was also considered possible. Further, competing hyper-arousal and hypo-arousal models of alexithymia have been proposed (Neumann, Sollers, Thayer \& Waldstein, 2004) and these would appear to make opposite predictions concerning the relationship of alexithymia to caffeine intake. That is, a hyperarousal model would logically predict caffeine avoidance by alexithymic individuals, whereas a hypo-arousal model would predict elevated caffeine intake by those with alexithymia.

The present study examined caffeine use in relation to alexithymia among young adult university students who are regular users of caffeine. University students have been reported to use caffeine at high levels, and self-reports of adverse caffeine-related anxiogenic effects are fairly common in this population (Bradley \& Petree, 1990). Indices of everyday frontal lobe related behavioural functioning, anxiety symptoms, sensitivity to punishment, sensitivity to reward, and alcohol use were also examined in relation to both caffeine use and alexithymia in an attempt to replicate previous findings with regard to trait correlates of both alexithymia and caffeine use. Punishment sensitivity was expected to be negatively related to caffeine use due to learned avoidance of the drug by chronically trait anxious or anxiety-sensitive individuals. Sensitivity to reward was speculatively predicted to be positively associated with caffeine use due to this trait's positive associations with use of alcohol and illicit drugs (Dawe \& Loxton, 2004; Genovese \& Wallace, 2007; Lyvers, Duff, Basch \& Edwards, 2012) as well as evidence that caffeine has mild rewarding properties (Hughes et al., 1993). The primary issue addressed by the present study was whether alexithymia would be positively related to use of caffeine, as it is for other psychoactive substances, or instead show a negative relationship due to caffeine's anxiogenic properties and consistent with a hyper-arousal interpretation of alexithymia. 


\section{Method}

\section{Participants}

All participants were currently enrolled in a course of study at a university in southeast Queensland, and were non-smokers between the ages of 18 and 30 years who at least occasionally consume caffeine. Further criteria included no use of illicit drugs within the past month, no current use of medication for a psychiatric or neurological illness, and never sustained a serious head injury. Recruitment was implemented via a sign-up sheet posted in the psychology department offering 1 credit point towards an undergraduate psychology subject for research participation, and also via direct approach to students on campus offering the incentive of a random draw for a $\$ 50$ gift voucher. The total number of participants who completed the research protocol was 120 , which was subsequently reduced to 106 after deletion of those who had missing data or were identified as multivariate outliers using Mahalanobis Distance. There were 39 males and 67 females in the final sample and their mean age was 21.21 years $(S D=3.90)$. Participants were assured of the anonymity of their data as per university human research ethics committee guidelines.

\section{Materials}

Demographics Questionnaire. A demographics questionnaire assessed participants' age, gender, marital status, educational level and employment status. For screening purposes participants were asked to indicate whether they had taken any illicit drugs within the past month, if they were smokers, if they had ever suffered a serious head injury, and if they were taking any medication for a psychiatric or neurological condition. Recent illicit drug users, smokers, those on psychiatric or neurological medication and those who reported having suffered a serious head injury were excluded from participation.

Caffeine Consumption Questionnaire (CCQ). The CCQ (Landrum, 1992) was developed in order to gauge the amount of caffeinated substances individuals consume daily. 
The CCQ lists beverages, chocolates and over the counter medications containing caffeine; adjacent to each item, the questionnaire specifies the average caffeine content per serving in milligrams. A participant's CCQ score was simply the sum of the typical quantity of caffeine ingested per day from all sources and servings in $\mathrm{mg}$.

Beck Anxiety Inventory (BAI). The widely used BAI (Beck \& Steer, 1993) lists 21 anxiety symptoms and asks participants to rate the severity of each symptom they experienced within the past week using a four point Likert scale ranging from 0 to $3(0=$ Not at all, $1=$ Mildly; it did not bother me much, 2 = Moderately; it was very unpleasant, but I could stand it, 3 = Severely; I could barely stand it). A total score is obtained by adding the ratings the respondent assigned to each question, producing an overall raw score that ranges between 0 and 63.

Sensitivity to Punishment and Sensitivity to Reward Questionnaire (SPSRQ). The SPSRQ (Torrubia, Avila, Molto \& Caseras, 2001) consists of 48 items that were conceptualised to represent two distinct biological dimensions of personality: the Behavioural Inhibition System (BIS), assessed by the Sensitivity to Punishment (SP) scale, and the Behavioural Approach System (BAS), assessed by the Sensitivity to Reward (SR) scale. SP and SR are represented by 24 items each. Participants respond to various situations using a dichotomous yes/no response format where responses are assigned a value of 1 for 'yes' or 0 for 'no,'; these are then summed to form a total score for SP and SR. SP has been found to be highly correlated with the Trait Anxiety scale of the State-Trait Anxiety Inventory (O’Connor, Colder \& Hawk, 2004; Torrubia et al, 2001).

Toronto Alexithymia Scale (TAS-20). The TAS-20 (Bagby, Parker \& Taylor, 1994; Parker, Taylor \& Bagby, 2003) is a 20 item self-report inventory that assesses the respondent's subjective ability to identify and describe their own emotions and their style of thinking. Participants respond to each statement using a 5-point scale, ranging from 1 to 5 ( 1 = Strongly 
Disagree, 2 = Moderately Disagree, 3 = Neither Disagree nor Agree, $4=$ Moderately Agree, and 5 = Strongly Agree). The TAS-20 exhibits a three factor structure corresponding to the three subscales, Difficulty Identifying Feelings (DIF), Difficulty Describing Feelings (DDF), and Externally-Oriented Thinking (EOT). A total score is obtained by adding the ratings the respondent assigns to each question, producing an overall score that can be used to identify those who are alexithymic (61 or greater), borderline alexithymic (52-60) or non-alexithymic (51 or less). Total scores were used for the purposes of the present study.

Frontal Systems Behaviour Scale (FrSBe). The FrSBe (Grace \& Malloy, 2001) consists of 46 items that were designed to measure behavioural consequences of injuries to the frontal lobes. The FrSBe asks participants to rate their everyday behaviour using a 5 point Likert scale ranging from 1 to $5(1=$ Almost Never, $2=$ Seldom, $3=$ Sometimes, $4=$ Frequently, 5 = Almost Always). When used as originally intended for brain-injured patients the FrSBe asks respondents to rate their behaviour 'before illness or injury' and 'at the present time'. In the present study only present time ratings were obtained, as in other research using the FrSBe in non-brain-injured individuals (e.g., Lyvers, Duff et al, 2012; Spinella, 2003; Verdejo-Garcia, Rivas-Pereza, Lopez-Torrecillasa, \& Perez-Garcia, 2006). The FrSBe has three subscales sensitive to damage to different prefrontal regions: Apathy (anterior cingulate, 14 items; e.g., 'Have difficulty starting an activity, lack initiative, motivation'), Executive Dysfunction (dorsolateral prefrontal cortex, 17 items; e.g., 'I repeat certain actions or get stuck on certain ideas'), and Disinhibition (orbitofrontal cortex, 15 items; e.g., 'I do things impulsively'). Factor analysis supports the three factor structure corresponding to the three subscales (Stout, Ready, Grace, Malloy \& Paulsen, 2003). Both total and subscale scores were examined in the present study.

Alcohol Use Disorders Identification Test (AUDIT). The AUDIT (Babor, De la Fuente, Saunders, \& Grant, 1992) is a widely used 10-item self-report screening tool for 
hazardous, harmful or dependent patterns of alcohol use. Items 1-3 refer to alcohol consumption (e.g., "How often do you have a drink containing alcohol?"), items 4-6 are related to alcohol dependence (e.g., "How often during the last year have you found that you were not able to stop drinking once you had started?"), and items 7-10 inquire about alcoholrelated harm or consequences (e.g., "Have you or someone else been injured because of your drinking?"). All items on the AUDIT are scored from 0-4 and can be summed to yield a total score ranging from 0 (non-drinkers) to 40 , with scores of 0-7 indicating low risk drinking, 8 15 hazardous drinking, and 16 or higher harmful drinking.

\section{Procedure}

After receiving ethics approval from the university's human research ethics committee, a participant signup sheet was posted on the research participation board in the psychology department, with an explanatory statement describing the study and the incentive of 1 credit point towards an undergraduate psychology subject. The signup sheet asked participants to provide their name and email address. Those who were interested in taking part in the study were contacted via email and asked to indicate a suitable time to meet on campus. Researchers met with participants at the arranged time, described the study and informed participants of the anonymity of their data. They were instructed not to provide any personally identifying information on any of the questionnaires. Participants were then handed a copy of the questionnaire battery and were asked to read the explanatory statement before completing the measures. Upon completion, the questionnaire battery was placed in a large envelope and participants were handed a credit slip for their participation. Other students were approached at random on campus and asked if they would like to participate in a research study for the incentive of entering a random draw for a $\$ 50$ gift voucher. Those who agreed underwent the same procedure as described above, except upon completion these participants were handed a raffle ticket. They wrote their name and contact phone number on the ticket, which was then 
placed in a separate envelope. Participants were informed that the raffle would take place after completion of all data collection and the winner would be contacted via telephone. After completion of all data collection, the winning raffle ticket was drawn and the winner provided with the $\$ 50$ gift voucher.

\section{Results}

Correlations. Intercorrelations were calculated among all continuous variables; these results are shown in Table 1. As can be seen in the table, daily caffeine intake in mg as estimated by CCQ was significantly positively correlated with scores on the BAI, TAS-20 and AUDIT. Other significant correlations were as expected based on previous work (Lyvers, Czerczyk, Follent \& Lodge, 2009; Lyvers, Duff \& Hasking, 2011; Lyvers, Duff et al., 2012): the positive correlations of SR with AUDIT and with FrSBe Disinhibition and Executive Dysfunction; TAS-20 with BAI, SP, AUDIT and all three FrSBe subscales; and AUDIT with SR, Disinhibition and Executive Dysfunction (see Table 1).

Group comparisons. Based on TAS-20 scores, $17 \%$ of the present Australian university student sample was defined as alexithymic, which is very close to the percentage defined as alexithymic by the same criterion in a sample of American university students as reported by Neumann et al. (2004). Using the standard TAS-20 cutoffs, alexithymic participants $(n=18)$ were distinguished from borderline $(n=14)$ and non-alexithymic participants $(n=74)$ for group comparison on the measures of interest using multivariate analysis of variance (MANOVA). ANOVA indicated that these groups did not significantly differ on age $(p=.09)$, and chi-square test indicated they did not significantly differ in gender composition $(p=.35)$; inclusion of age and gender as covariates in the MANOVA did not alter the findings. Box's M Test was not significant $(p=.10)$, and Levene's Test of Equality of Error Variances was significant only for CCQ $(p=.02)$ despite unequal sample sizes. A more stringent alpha criterion of $p<.01$ was thus used for CCQ (Tabachnik \& Fidell, 2007). 
MANOVA comparing the three alexithymia groups on CCQ, BAI, SP, SR, and total FrSBe scores showed an overall significant multivariate effect according to Pillai's Trace, $F(10$, $200)=4.30, p<.0001$, partial $\eta^{2}=.18$, observed power $=1.00$. Univariate effects were significant for all dependent measures: CCQ, $F(2,103)=4.93, p=.009$, partial $\eta^{2}=.09$, observed power $=.80 ;$ BAI, $F(2,103)=7.23, p=.001$, partial $\eta^{2}=.12$, observed power $=$ .93; SP, $F(2,103)=5.95, p=.004$, partial $\eta^{2}=.10$, observed power $=.87 ; \mathrm{SR}, F(2,103)=$ $3.92, p=.02$, partial $\eta^{2}=.07$, observed power $=.70$; and $\operatorname{FrSBe}, F(2,103)=7.33, p=.001$, partial $\eta^{2}=.13$, observed power $=.93$. The conservative Tukey post hoc test revealed that alexithymics scored significantly higher than non-alexithymics on all dependent measures except SR and significantly higher than borderline alexithymics on FrSBe, with borderline alexithymics scoring significantly higher than non-alexithymics on SP (see Table 3 for the relevant means). The high daily caffeine consumption reported by alexithymics was a surprising result meriting further investigation.

Regression analysis. To examine TAS-20 as a potential predictor of caffeine use after controlling for all other variables, a sequential hierarchical regression was conducted with CCQ scores as the criterion. Age, gender, AUDIT, SP, SR, BAI, and total FrSBe scores were entered at step 1, followed by TAS-20 scores at step 2. At step 1 the model was not significant, $R=.34, R^{2}=.12, F(7,93)=1.73, p=.11$. Only with the addition of TAS-20 scores at step 2 did the model become significant, $R=.43, R^{2}=.18, F$ change $(1,92)=7.70$, $p=.007$. Table 2 displays the unstandardised regression coefficients (B), standardised regression coefficients $(\beta), t$ scores and the $R^{2}$ change for all variables at each step. Only TAS-20 and SP scores predicted caffeine consumption as measured by CCQ, with TAS-20 a positive predictor and SP a negative predictor.

\section{Discussion}


Perhaps surprisingly, alexithymics reported consuming nearly twice as much caffeine per day on average compared to non-alexithymic controls or those with borderline alexithymia. This was contrary to predictions based on the association of anxiety with alexithymia as well as the hyper-arousal model of alexithymia. Other results were generally consistent with expectations. Scores on the BAI, which asks about anxiety symptoms experienced over the past week, were positively related to CCQ caffeine consumption, consistent with an anxiogenic effect of caffeine at higher intake levels (Bradley \& Petree, 1990; Evans \& Griffith, 1992; Lara, 2010; Lieberman et al., 1987; Penolazzi et al, 2012). Although the BAI and the SP scale of the SPSRQ were positively correlated as expected, the predicted negative relationship between SP and caffeine consumption only emerged after TAS-20 alexithymia scores were taken into account in the regression model. Other relationships were consistent with predictions based on previous research in similar samples, such as the significant positive associations between AUDIT, SR, and the Disinhibition and Executive Dysfunction scales of the FrSBe, and of TAS-20 with AUDIT and all three FrSBe subscales. MANOVA revealed that alexithymics as defined by TAS-20 scored significantly higher than non-alexithymics on the BAI, SP, and FrSBe, consistent with predictions.

The observed positive relationship between alexithymia and intake of caffeine, a drug with known anxiogenic properties, may seem surprising when one considers the association of alexithymia with trait anxiety and anxiety sensitivity (Berthoz et al., 1999; DeGucht et al., 2004; de Timary et al., 2008), as evidenced by the moderate positive correlation of TAS-20 alexithymia scores with SP scores in the present study. Given the anxiogenic effects of higher caffeine doses, and the high trait anxiety and anxiety sensitivity of alexithymics, a negative relationship between alexithymia and caffeine consumption may seem most plausible based on the assumption that anxiety is an aversive state that those with alexithymia would attempt to minimize by avoiding higher doses of caffeine. Instead the present results indicate that 
those with alexithymia tended to consume caffeine much more heavily than those without alexithymia, similar to their heavier reported use patterns for alcohol and illicit drugs (Lyvers et al., in press; Thorberg et al., 2009). Lyvers, Hasking et al. (2012) found a positive relationship between the TAS-20 and coping motives for drinking alcohol that was mediated by SP, suggesting that alexithymics tend to use alcohol to alleviate anxiety and stress. In the present study, TAS-20 scores were positively related to both AUDIT and SP scores, suggesting that one possible explanation for the positive association between TAS-20 and caffeine consumption - which was also positively associated with AUDIT - is that alexithymic tendencies promote heavier drinking, which in turn may promote heavier caffeine consumption in an attempt to alleviate adverse after-effects of alcohol. This post hoc idea was explored using a simple path analysis to see if AUDIT mediated the relationship between TAS-20 and CCQ caffeine consumption, however there was no evidence of mediation (Sobel Test $p=.16$, n.s.). Thus although the heavy caffeine intake of alexithymics in the present study fits with heavier use of other substances by those with alexithymia as shown in previous research, the motives for such use are unclear.

Another potential clue arises from the strong associations between TAS-20 alexithymia scores and all three FrSBe frontal lobe dysfunction subscales in the present study. Perhaps the cognitive dysfunction reported by university students with alexithymia motivates them to consume more caffeine for its cognitive enhancing properties (Van Duinen et al., 2005). Indeed, a positive effect of caffeine on performance of the Wisconsin Card Sorting Test - a widely used neuropsychological test of frontal lobe related executive cognitive functioning - has been reported (Lyvers, Brooks \& Matica, 2004). However neither the FrSBe nor any of its subscales were related to caffeine consumption in the present study. The most plausible interpretation of the present findings is that heavy caffeine consumption by those with alexithymia is consistent with the hypo-arousal model of alexithymia (see 
Neumann et al., 2004), according to which alexithymics are characterized by low autonomic arousal. Perhaps those with alexithymia consume caffeine more heavily than nonalexithymics in an attempt to optimize inherently low arousal levels (Anderson \& Revelle, 1994). Such an interpretation of the present results is necessarily tentative, however. Limitations of the present study include the cross-sectional design and university student sample. Further research in larger, more representative samples is warranted to replicate and extend the present finding of heavy caffeine use by university students with alexithymia and to determine whether the observed positive relationship between alexithymia and caffeine use also occurs in non-student and older samples. A longitudinal approach would also be helpful in elucidating the stability of alexithymia over time and its relationship to caffeine and other drug use. Reliance on a self-report scale (TAS-20) to measure alexithymia, a condition where some aspects of introspective self-awareness are limited, is another potential issue that could be addressed by using clinician ratings in addition to TAS20 to assess alexithymia; interestingly however, TAS-20 has been reported to have better predictive utility than observer ratings of alexithymia in a substance dependent sample (Thorberg et al., 2010). Finally, a recent Brazilian study (Lara et al., 2011) has suggested that palatability of cola drinks may account for some of the links between personality and caffeine consumption rather than caffeine per se, however this interpretation is not relevant to the present findings as only $7 \%$ of caffeine intake in the present sample was in the form of cola drinks.

In conclusion, present findings indicate that people with alexithymia report considerably heavier caffeine use than do those without alexithymia, similar to the positive associations of alexithymia with use of alcohol and other substances as shown by previous research. The mechanisms underlying such relationships are however unclear and warrant further investigation. 
Alexithymia and caffeine 14

\section{References}

Anderson, K.J., \& Revelle, W. (1994). Impulsivity and time of day: Is rate of change in arousal a function of impulsivity? Journal of Personality and Social Psychology, 67,

This is an Accepted Manuscript version of the following article:

Lyvers, M., Duric, N., \& Thorberg, F. A. (2014). Caffeine use and alexithymia in university students. Journal of Psychoactive Drugs, 46(4), 340-346. https://doi.org/10.1080/02791072.2014.942043.

It is deposited under the terms of the Creative Commons Attribution-NonCommercial-NoDerivatives License, which permits non-commercial re-use, distribution, and reproduction in any medium, provided the original work is properly cited, and is not altered, transformed, or built upon in any way. 
334-344.

Babor, T. F., De la Fuente, J. R., Saunders, J., \& Grant, M. (1992). AUDIT: The Alcohol Use Disorders Identification Test. Geneva: World Health Organization.

Bagby, R. M., Parker, J. D., \& Taylor, G. J. (1994). The twenty-item Toronto Alexithymia Scale: Item selection and cross-validation of the factor structure. Journal of Psychosomatic Research, 38, 23-32.

Bagby, R. M., Taylor, G. J., \& Parker, J. D. A. (1994). The twenty-item Toronto Alexithymia Scale: II. Convergent, discriminant, and concurrent validity. Journal of Psychosomatic Research, 38, 33-40.

Beck, A.T., \& Steer, R.A. (1993). Beck Anxiety Inventory (BAI) Manual. San Antonio: Psychological Corporation.

Berthoz, S., Consoll, S., Perez-Diaz, F., \& Jouvent, R. (1999). Alexithymia and anxiety: Compounded relationship? A psychometric study. European Psychiatry, 14, 372-378. Bradley, J.R., \& Petree, A. (1990). Caffeine consumption, expectancies of caffeine-enhanced performance, and caffeinism symptoms among university students. Journal of Drug Education, 20, 319-328.

Bryan, J. (2008). Psychological effects of dietary components of tea: Caffeine and Ltheanine. Nutrition Reviews, 66, 82-90.

Childs, E., \& De Wit, H. (2006). Subjective, behavioural, and psychological effects of acute caffeine in light, non-dependent caffeine users. Psychopharmacology, 185, 514-523.

Dawe, S. \& Loxton, N. J. (2004). The role of impulsivity in the development of substance use and eating disorders. Neuroscience and Biobehavioural Reviews, 28, 343-351.

De Gucht, V., Fischler, B., \& Heiser, W. (2004). Neuroticism, alexithymia, negative affect, and positive affect as determinants of medically unexplained symptoms. Personality and Individual Differences, 36, 1655-1667. 
de Timary, P., Emmanuel, R., Luminet, O., Fillee, C., \& Mikolajczak, M. (2008).

Relationship between alexithymia, alexithymia factors and salivary cortisol in men exposed to a social stress test. Psychoneuroendocrinology, 33, 1160-1164.

Devine, H., Stewart, S.H., \& Watt, M.C. (1999). Relations between anxiety sensitivity and dimensions of alexithymia. Journal of Psychosomatic Research, 47, 145-158.

Evans, S.M., \& Griffiths, P.R. (1992). Caffeine tolerance and choice in humans. Psychopharmacology, 108, 51-59.

Franz, M., Popp, K., Schaefer, R., Sitte, W., Schneider, C., Hardt, J., Decker, O., \& Braeler, E. (2008). Alexithymia in the German general population. Social Psychiatry and Psychiatric Epidemiology, 43, 54-62.

Genovese, J., \& Wallace, D. (2007). Reward sensitivity and substance abuse in middle school and high school students. Journal of Genetic Psychology, 168, 465-469.

Grace, J., \& Malloy, P. F. (2001). Frontal Systems Behavior Scale. Professional manual.

Lutz, FL: Psychological Assessment Resources, Inc.

Grilly, D.M. (2006). Drugs and human behavior. Boston: Pearson.

Hughes, J.R., Oliverto, A.H., Helzer, J.E., Higgins, S.T., \& Bickel, W.K. (1992). Should caffeine abuse, dependence, or withdrawal be added to DSM-IV and ICD-10? American Journal of Psychiatry, 149, 33-40.

Kauhanen, J., Julkunen, J., \& Salonen, J.T. (1992). Coping with inner feelings and stress: Heavy alcohol use in the context of alexithymia. Behavioral Medicine, 18, 121-126.

Landrum, R.E. (1992). College students' use of caffeine and its relationship to personality. College Student Journal, 26, 151-155.

Lara, D.R. (2010). Caffeine, mental health, and psychiatric disorders. Journal of Alzheimer's Disease, 20, S239-S248. 
Lara, D.R., Antoniolli, E., Frozi, J., Schneider, R., \& Ottoni, G.L. (2011). Distinct personality traits associated with intake of coffee, tea, and cola drinks and smoking. Journal of Caffeine Research, 1, 101-108.

Lieberman, H.R., Wurtman, R.J., Emde, G.G., Roberts, C., \& Covielle, I.L.G. (1987). The effects of low doses of caffeine on human performance and mood. Psychopharmacology, 92, 308-312.

Lyvers, M., Brooks, J., \& Matica, D. (2004). Effects of caffeine on cognitive and autonomic measures in heavy and light caffeine users. Australian Journal of Psychology, 56, $33-41$.

Lyvers, M., Czerczyk, C., Follent, A., \& Lodge, P. (2009). Disinhibition and reward sensitivity in relation to alcohol consumption by university students. Addiction Research \& Theory, 17, 668-677.

Lyvers, M., Duff, H., \& Hasking, P. (2011). Risky alcohol use and age at onset of regular alcohol consumption in relation to frontal lobe indices, reward sensitivity and rash impulsiveness. Addiction Research \& Theory, 19, 251-259.

Lyvers, M., Duff, H., Basch, V., \& Edwards, M. (2012). Influences of rash impulsiveness and reward sensitivity on risky drinking in university students: Evidence of mediation by frontal systems. Addictive Behaviors, 37, 940-946.

Lyvers, M., Hasking, P., Albrecht, B., \& Thorberg, F.A. (2012). Alexithymia and alcohol: The roles of punishment sensitivity and drinking motives. Addiction Research \& Theory, 20, 348-357.

Lyvers, M., Jamieson, R., \& Thorberg, F.A. Risky cannabis use is associated with alexithymia, frontal lobe dysfunction and impulsivity in young adult cannabis users. Journal of Psychoactive Drugs, in press.

Lyvers, M., Onuoha, R., Thorberg, F.A., \& Samios, C. (2012). Alexithymia in relation to 
parental alcoholism, everyday frontal lobe functioning and alcohol consumption in a non-clinical sample. Addictive Behaviors, 37, 205-210.

Nemiah, J. C., Freyberger, H., \& Sifneos, P. E. (1976). Alexithymia: A view of the psychosomatic process. In O. Hill (Ed.), Modern trends in psychosomatic medicine (vol. 3; pp. 430-439). London: Butterworths.

Neumann, S.A., Sollers III, J.J., Thayer, J.F., \& Waldstein, S.R. (2004). Alexithymia predicts attenuated autonomic reactivity, but prolonged recovery to anger recall in young women. International Journal of Psychophysiology, 53, 183-195.

O’Connor, R.M., Colder, C.R., \& Hawk, L.W. (2004). Confirmatory factor analysis of the sensitivity to punishment and sensitivity to reward questionnaire. Personality and Individual Differences, 37, 985-1002.

Parker, J. D. A., Taylor, G. J., \& Bagby, R. M. (2003). The 20-Item Toronto Alexithymia Scale III: Reliability and factorial validity in a community population. Journal of Psychosomatic Research, 55, 269-275.

Penolazzi, B., Natale, V., Leone, L., \& Russo, P.M. (2012). Individual differences affecting caffeine intake. Analysis of consumption behaviours for different times of day and caffeine sources. Appetite, 58, 971-977.

Read, J.P., Wood, M.D., Kahler, C.W., Maddock, J.E., \& Palfai, T.P. (2003). Examining the role of drinking motives in college student alcohol use and problems. Psychology of Addictive Behaviors, 17, 13-23.

Smith, A. (2002). Effects of caffeine on human behaviour. Food and Chemical Toxicology, $40,1243-1255$.

Spinella, M. (2003). Relationship between drug use and prefrontal-associated traits. Addiction Biology, 8, 67-74. 
Stewart, S., Zvolensky, M., \& Eifert, G. (2002). The relations of anxiety sensitivity, experiential avoidance, and alexithymic coping to young adults' motivations for drinking. Behavior Modification, 26, 274-296.

Stout, J. C., Ready, R. E., Grace, J., Malloy, P. F., \& Paulsen, J. S. (2003). Factor analysis of the frontal systems behaviour scale (FrSBe). Assessment, 10, 79-85.

Tabachnick, B. G., \& Fidell, L. S. (2007). Using Multivariate Statistics (Fifth Edition). Boston: Allyn and Bacon.

Telch, M. J., Silverman, A., \& Schmidt, N. B. (1996). Effects of anxiety sensitivity and perceived control on emotional responding to caffeine challenge. Journal of Anxiety Disorders, 10, 21-35.

Thorberg, F.A., Young, R., Sullivan, K.A., \& Lyvers, M. (2009). Alexithymia and alcohol use disorders: A critical review. Addictive Behaviors, 34, 237-245.

Thorberg, F. A., Young, R. McD., Sullivan, K. A., Lyvers, M., Connor, J. P., \& Feeney, GFX. (2010). A psychometric comparison of the Toronto Alexithymia Scale (TAS20 ) and the Observer Alexithymia Scale (OAS) in an alcohol dependent sample. Personality \& Individual Differences, 43, 119-123.

Torrubia, R., Avila, C., Molto, J., \& Caseras, X. (2001). The Sensitivity to Punishment and Sensitivity to Reward Questionnaire (SRSPQ) as a measure of Gray's anxiety and impulsivity dimensions. Personality and Individual Differences, 31, 837-862.

Troisi, A., Pasini, A., Saracco, M., \& Spalletta, G. (1998). Psychiatric symptoms in male cannabis users not using other illicit drugs. Addiction, 93, 487-492.

Tutkun, H., Savas, H.A., Zoroglu, S., Esgi, K., Herken, H., \& Tiryaki, N. (2004).

Relationship between alexithymia, dissociation and anxiety in psychiatric outpatients from Turkey. Israel Journal of Psychiatry and Related Sciences, 41, 118-124. 
Van Duinen, H., Lorist, M.M., \& Zijdewind, I. (2005). The effect of caffeine on cognitive task performance and motor fatigue. Psychopharmacology, 180, 539-547.

Verdejo-Garcia, A., Rivas-Pereza, C., Lopez-Torrecillasa, F., \& Perez-Garcia, M. (2006). Differential impact of severity of drug use on frontal behavioural symptoms. Addictive Behaviours, 31, 1373-1382. 
Table 1

Correlations among study variables

\begin{tabular}{llllllllll}
\hline Age & CCQ & BAI & SP & SR & TAS & Apathy & Disin & ExeDys & AUDIT \\
\hline
\end{tabular}

\begin{tabular}{|c|c|c|c|c|c|c|c|c|c|}
\hline Age & 1 & & & & & & & & \\
\hline CCQ & .01 & 1 & & & & & & & \\
\hline BAI & -.08 & $.21 *$ & 1 & & & & & & \\
\hline SP & -.05 & -.09 & $.30 * *$ & 1 & & & & & \\
\hline SR & -.10 & $.19 *$ & $.28 * *$ & .09 & 1 & & & & \\
\hline TAS & $-.31 * *$ & $.26^{* *}$ & $.39 * *$ & $.35^{* *}$ & .17 & 1 & & & \\
\hline Apathy & -.16 & .02 & $.25^{* *}$ & $.36^{* *}$ & .11 & $.39 * *$ & 1 & & \\
\hline Disin & $-.29 * *$ & .11 & $.30 * *$ & .09 & $.52 * *$ & $.37 * *$ & $.46^{* *}$ & 1 & \\
\hline ExeDys & -.15 & .03 & $.25 * *$ & $.20 *$ & $.35^{* *}$ & $.37 * *$ & $.70 * *$ & $.68 * *$ & 1 \\
\hline AUDIT & $-.19^{*}$ & $.21 *$ & .18 & .05 & $.36^{* *}$ & $.20^{*}$ & .11 & $.34 * *$ & $.21 * *$ \\
\hline
\end{tabular}

Note. $\mathrm{CCQ}=$ Caffeine Consumption Questionnaire; BAI = Beck Anxiety Inventory; SP = Sensitivity to Punishment; SR = Sensitivity to Reward; TAS $=$ Toronto Alexithymia Scale; Disin = Disinhibition; ExeDys = Executive Dysfunction; AUDIT = Alcohol Use Disorders Identification Test. $* p<.05 * * p<.01$ 
Table 2

Regression Coefficients of Predictors of Caffeine Consumption

\begin{tabular}{|c|c|c|c|c|c|}
\hline & Variable & $B$ & $\beta$ & $t$ & $R_{\text {change }}^{2}$ \\
\hline \multirow[t]{7}{*}{ Step 1} & Age & 2.04 & .03 & .25 & \\
\hline & Gender & -52.69 & -.09 & -.78 & \\
\hline & AUDIT & 4.79 & .09 & .83 & \\
\hline & BAI & 5.62 & .20 & 1.76 & \\
\hline & SP & -10.44 & -.19 & -1.73 & \\
\hline & SR & 9.23 & .14 & 1.21 & \\
\hline & FrSBe & -1.69 & -.12 & -.97 & .12 \\
\hline \multirow[t]{8}{*}{ Step 2} & Age & 7.76 & .10 & .96 & \\
\hline & Gender & -47.80 & -.08 & -.73 & \\
\hline & AUDIT & 3.60 & .07 & .63 & \\
\hline & BAI & 3.83 & .14 & 1.21 & \\
\hline & SP & -14.39 & -.26 & $-2.39 *$ & \\
\hline & SR & 11.64 & .17 & 1.57 & \\
\hline & FrSBe & -3.00 & -.20 & -1.72 & \\
\hline & TAS & 7.60 & .32 & $2.77 * *$ & .07 \\
\hline
\end{tabular}


Table 3

Means and Standard Deviations for Alexithymia, Borderline Alexithymia and NonAlexithymia Groups on Trait and Mood Indices (see text for definitions of measures)

\begin{tabular}{|c|c|c|c|}
\hline Measure & Group & $M$ & $S D$ \\
\hline \multirow[t]{3}{*}{ Caffeine $(\mathrm{mg})^{* *}$} & Alexithymia & 503.06 & 430.04 \\
\hline & Borderline & 274.29 & 204.29 \\
\hline & Control & 275.09 & 246.48 \\
\hline \multirow[t]{3}{*}{ BAI** } & Alexithymia & 17.22 & 11.69 \\
\hline & Borderline & 10.86 & 6.31 \\
\hline & Control & 7.59 & 9.71 \\
\hline \multirow[t]{3}{*}{ SP** } & Alexithymia & 12.44 & 5.24 \\
\hline & Borderline & 13.57 & 5.57 \\
\hline & Control & 9.28 & 4.96 \\
\hline \multirow[t]{3}{*}{$\mathrm{SR}^{*}$} & Alexithymia & 13.78 & 4.21 \\
\hline & Borderline & 9.64 & 4.14 \\
\hline & Control & 11.51 & 4.23 \\
\hline \multirow[t]{3}{*}{$\mathrm{FrSBe}^{* *}$} & Alexithymia & 114.78 & 16.08 \\
\hline & Borderline & 95.86 & 17.52 \\
\hline & Control & 95.85 & 20.01 \\
\hline
\end{tabular}

$* p<.05 \quad * * p<.01$ 